\title{
Acquired Hydrocephalus
}

National Cancer Institute

\section{Source}

National Cancer Institute. Acquired Hydrocephalus. NCI Thesaurus. Code C98803.

Hydrocephalus that results from head trauma, brain tumors, intracranial hemorrhage, or meningitis. 